\title{
Multi-frequency Phase Unwrapping from Noisy Data: Adaptive Local Maximum Likelihood Approach
}

\author{
José Bioucas-Dias $^{1}$, Vladimir Katkovnik ${ }^{2}$, Jaakko Astola ${ }^{2}$, \\ and Karen Egiazarian ${ }^{2}$ \\ 1 Instituto de Telecomunicações, Instituto Superior Técnico, TULisbon, \\ 1049-001 Lisboa, Portugal \\ bioucas@lx.it.pt \\ 2 Signal Processing Institute, University of Technology of Tampere, \\ P.O. Box 553, Tampere, Finland \\ \{katkov,jta, karen\}@cs.tut.fi
}

\begin{abstract}
The paper introduces a new approach to absolute phase estimation from frequency diverse wrapped observations. We adopt a discontinuity preserving nonparametric regression technique, where the phase is reconstructed based on a local maximum likelihood criterion. It is shown that this criterion, applied to the multifrequency data, besides filtering the noise, yields a $2 \pi Q$-periodic solution, where $Q>1$ is an integer. The filtering algorithm is based on local polynomial (LPA) approximation for the design of nonlinear filters (estimators) and the adaptation of these filters to the unknown spatially smoothness of the absolute phase. Depending on the value of $Q$ and of the original phase range, we may obtain complete or partial phase unwrapping. In the latter case, we apply the recently introduced robust (in the sense of discontinuity preserving) PUMA unwrapping algorithm 1. Simulations give evidence that the proposed method yields state-of-the-art performance, enabling phase unwrapping in extraordinary difficult situations when all other algorithms fail.
\end{abstract}

Keywords: Interferometric imaging, phase unwrapping, diversity, local maximum-likelihood, adaptive filtering.

\section{Introduction}

Many remote sensing systems exploit the phase coherence between the transmitted and the scattered waves to infer information about physical and geometrical properties of the illuminated objects such as shape, deformation, movement, and structure of the object's surface. Phase estimation plays, therefore, a central role in these coherent imaging systems. For instance, in synthetic aperture radar interferometry (InSAR), the phase is proportional to the terrain elevation height; in magnetic resonance imaging, the phase is used to measure temperature, to map the main magnetic field inhomogeneity, to identify veins in the tissues, and to segment water from fat. Other examples can be found in adaptive optics, 
diffraction tomography, nondestructive testing of components, and deformation and vibration measurements (see, e.g., [2], 4], 3], 5]). In all these applications, the observation mechanism is a $2 \pi$-periodic function of the true phase, hereafter termed absolute phase. The inversion of this function in the interval $[-\pi, \pi)$ yields the so-called principal phase values, or wrapped phases, or interferogram; if the true phase is outside the interval $[-\pi, \pi)$, the associated observed value is wrapped into it, corresponding to the addition/subtraction of an integer number of $2 \pi$. It is thus impossible to unambiguously reconstruct the absolute phase, unless additional assumptions are introduced into this inference problem.

Data acquisition with diversity has been exploited to eliminate or reduce the ambiguity of absolute phase reconstruction problem. In this paper, we consider multichannel sensors, each one operating at a different frequency (or wavelengths).

Let $\psi_{s}$, for $s=1, \ldots, L$, stand for the wrapped phase acquired by a $L$-channel sensor. In the absence of noise, the wrapped phase is related with the true absolute phase, $\varphi$, as $\mu_{s} \varphi=\psi_{s}+2 \pi k_{s}$, where $k_{s}$ is an integer, $\psi_{s} \in[-\pi, \pi)$, and $\mu_{s}$ is a channel depending scale parameter, to which we attach the meaning of relative frequency. This parameter establishes a link between the absolute phase $\varphi$ and the wrapped phase $\psi_{s}$ measured at the $s$-channel:

$$
\psi_{s}=W\left(\mu_{s} \varphi_{s}\right) \equiv \bmod \left\{\mu_{s} \varphi+\pi, 2 \pi\right\}-\pi, \quad s=1, \ldots, L,
$$

where $W(\cdot)$ is the so-called wrapping operator, which decomposes the absolute phase $\varphi$ into two parts: the fractional part $\psi_{s}$ and the integer part defined as $2 \pi k_{s}$. The integers $k_{s}$ are known in interferometry as fringe orders. We assume that the frequencies for the different channels are strictly decreasing, i.e., $\mu_{1}>$ $\mu_{2}>\ldots>\mu_{L}$, or, equivalently, the corresponding wavelengths $\lambda_{s}=1 / \mu_{s}$ are strictly increasing, $\lambda_{1}<\lambda_{2}, \ldots \lambda_{L}$.

Let us mention some of the techniques used for the multifrequency phase unwrap. Multi-frequency interferometry (see, e.g., [16) provides a solution for fringe order identification using the method of excess fractions. This technique computes a set of integers $k_{s}$ compatible with the simultaneous set of equations $\mu_{s} \varphi=\psi_{s}+2 \pi k_{s}$, for $s=1, \ldots, L$. It is assumed that the frequencies $\mu_{s}$ do not share common factors, i.e., they are pair-wise relatively prime. The solution is obtained by maximizing the interval of possible absolute phase values.

A different approach formulates the phase unwrapping problem in terms of the Chinese remainder theorem, where the absolute phase $\varphi$ is reconstructed from the remainders $\psi_{s}$, given the frequencies $\mu_{s}$. This formulation assumes that all variables known and unknown are scaled to be integral. An accurate theory and results, in particular concerning the existence of a unique solution, is a strong side of this approach [18.

The initial versions of the excess fraction and Chinese remainder theorem based methods are highly sensitive to random errors. Efforts have been made to make these methods resistant to noise. The works [19] and 17], based on he Chinese remainder approach, are results of these efforts.

Statistical modeling for multi-frequency phase unwrapping based on the maximum likelihood approach is proposed in [13. This work addresses the surface 
reconstruction from the multifrequency InSAR data. The unknown surface is approximated by local planes. The optimization problem therein formulated is tackled with simulated annealing.

An obvious idea that comes to mind to attenuate the damaging effect of the noise is prefiltering the wrapped observations. We would like, however, to emphasize that prefiltering, although desirable, is a rather delicate task. In fact, if prefiltering is too strong, the essential pattern of the absolute phase coded in the wrapped phase is damaged, and the reconstruction of absolute phase is compromised. On the other hand, if we do not filter, the unwrapping may be impossible because of the noise. A conclusion is, therefore, that filtering is crucial but should be designed very carefully. One of the ways to ensure efficiency is to adapt the strength of the prefiltering according to the phase surface smoothness and the noise level. In this paper, we use the wrapped phase prefiltering technique developed in 20] for a single frequency phase unwrapping.

\section{Proposed Approach}

We introduce a novel phase unwrapping technique based on local polynomial approximation (LPA) with varying adaptive neighborhood used in reconstruction. We assume that the absolute phase is a piecewise smooth function, which is well approximated by a polynomial in a neighborhood of the estimation point. Besides the wrapped phase, also the size and possibly the shape of this neighborhood are estimated. The adaptive window selection is based on two independent ideas: local approximation for design of nonlinear filters (estimators) and adaptation of these filters to the unknown spatially varying smoothness of the absolute phase. We use LPA for approximation in a sliding varying size window and intersection of confidence intervals (ICI) for window size adaptation. The proposed technique is a development of the PEARLS algorithm proposed for the single wavelength phase reconstruction from noisy data [20].

We assume that the frequencies $\mu_{s}$ can be represented as ratios

$$
\mu_{s}=p_{s} / q_{s}
$$

where $p_{s}, q_{s}$ are positive integers and the pairs $\left(p_{s}, q_{t}\right)$, for $s, t \in\{1, \ldots, L\}$ do not have common multipliers, i.e., $p_{s}$ and $q_{t}$ are pair-wise relatively prime.

Let

$$
Q=\prod_{s=1}^{L} q_{s} .
$$

Based on the LPA of the phase, the first step of the proposed algorithm computes the maximum likelihood estimate of the absolute phase. As a result, we obtain an unambiguous absolute phase estimates in the interval $[-Q \cdot \pi, Q$. $\pi)$. Equivalently, we get an $2 \pi Q$ periodic estimate. The adaptive window size LPA is a key technical element in the noise suppression and reconstruction of this wrapped $2 \pi Q$-phase. The complete unwrapping is achieved by applying an unwrapping algorithm. In our implementation, we use the PUMA algorithm [1, 
which is able to preserve discontinuities by using graph cut based methods to solve the integer optimization problem associated to the phase unwrapping.

The polynomial modeling is a popular idea for both wrapped phase denoising and noisy phase unwrap. Using the local polynomial fit in terms of the phase tracking for the phase unwrap is proposed in the paper [12]. In the paper [13] the linear local polynomial approximation of height profiles is used for the surface reconstruction from the multifrequency InSAR data. Different modifications of the local polynomial approximation oriented to wrapped phase denoising are introduced in the regularized phase-tracking [14, 15], the multiple-parameter least squares [8], and the windowed Fourier ridges [9]. Compared with these works, the efficiency of the PEARLS algorithm [20] is based on the window size selection adaptiveness introduced by the ICI technique, which locally adapts the amount of smoothing according to the data. In particular, the discontinuities are preserved, what is a sine quo non condition for the success of the posterior unwrapping; in fact, as discussed in [7, it is preferable to unwrap the noisy interferogram than a filtered version in which the discontinuities or the areas of high phase rate have been washed out. In this paper, the PEARLS [20] adaptive filtering is generalized for the multifrequency data. Experiments based on simulations give evidence that the developed unwrapping is very efficient for the continuous as well as discontinuous absolute phase with a range of the phase variation so large that there no alternative algorithms able to unwrap this data.

\section{Local Maximum Likelihood Technique}

Herein, we adopt the complex-valued $(\cos / \sin )$ observation model

$$
u_{s}=B_{s} \exp \left(j \mu_{s} \varphi\right)+n_{s}, s=1, \ldots, L, \quad B_{s} \geq 0,
$$

where $B_{s}$ are amplitudes of the harmonic phase functions, and $n_{s}$ is zero-mean independent complex-valued circular Gaussian random noises of variance equal to 1 , i.e., $E\left\{\operatorname{Re} n_{s}\right\}=0, E\left\{\operatorname{Im} n_{s}\right\}=0, E\left\{\operatorname{Re} n_{s} \cdot \operatorname{Im} n_{s}\right\}=0, E\left\{\left(\operatorname{Re} n_{s}\right)^{2}\right\}=1 / 2$, $E\left\{\left(\operatorname{Im} n_{s}\right)^{2}\right\}=1 / 2$. We assume that the amplitudes $B_{s}$ are non-negative in order to avoid ambiguities in the phase $\mu_{s} \varphi$, as the change of the amplitude sign is equivalent to a phase change of $\pm \pi$ in $\mu_{s} \varphi$. We note that the assumption of equal noise variance for all channel is not limitative as different noise variances can be accounted for by rescaling $u_{s}$ and $A_{s}$ in (4) by the corresponding noise standard deviation.

Model (4) accurately describes the acquisition mechanism of many interferometric applications, such as InSAR and magnetic resonance imaging. Furthermore, it retains the principal characteristics of most interferometric applications: it is a $2 \pi$-periodic function of $\mu_{s} \varphi$ and, thus, we have only access to the wrapped phase.

Since we are interested in two-dimensional problems, we assume that the observations are given on a regular $2 D$ grid, $X \subset \mathbb{Z}^{2}$. The unwrapping problem is to reconstruct the absolute phase $\varphi(x, y)$ from the observed wrapped noisy $\psi_{s}(x, y)$, for $x, y \in X$. 
Let us define the parameterized family of first order polynomials

$$
\tilde{\varphi}(u, v \mid \mathbf{c})=p^{T}(u, v) \mathbf{c},
$$

where $p=\left[p_{1}, p_{2}, p_{3}\right]^{T}=[1, u, v]^{T}$ and $\mathbf{c}=\left[c_{1}, c_{2}, c_{3}\right]^{T}$ is a vector of parameters. Assume that in some neighborhood of the point $(x, y)$, the phase $\varphi$ is well approximated by an element of the family (5); i.e., for $\left(x_{l}, y_{l}\right)$ in a neighborhood of the origin, there exists a vector $\mathbf{c}$ such that

$$
\varphi\left(x+x_{l}, y+x_{l}\right) \simeq \tilde{\varphi}\left(x_{l}, y_{l} \mid \mathbf{c}\right) .
$$

To infer $\mathbf{c}$ and $\mathbf{B} \equiv\left\{B_{1}, \ldots, B_{L}\right\}$ (see (4) ), we compute

$$
\hat{\mathbf{c}}=\arg \min _{\mathbf{c}, \mathbf{B} \geq \mathbf{0}} L_{h}(\mathbf{c}, \mathbf{B}) \text {. }
$$

where $L_{h}(\mathbf{c}, \mathbf{B})$ is a negative local log-likelihood function given by

$$
\begin{aligned}
& L_{h}(\mathbf{c}, \mathbf{B})= \\
& \sum_{s} \frac{1}{\sigma_{s}^{2}} \sum_{l} w_{h, l, s} \mid u_{s}\left(x+x_{l}, y+y_{l}\right)-B_{s} \exp \left(\left.j \mu_{s} \tilde{\varphi}\left(x_{l}, y_{l} \mid \mathbf{c}\right)\right|^{2} .\right.
\end{aligned}
$$

Terms $w_{h, l, s}$ are window weights and can be different for different channels.

The local model $\tilde{\varphi}(u, v \mid \mathbf{c})$ (5) is the same for all frequency channels. We start by minimization $L_{h}$ with respect to $\mathbf{B}$, which reduces to decoupled minimizations with respect to $B_{s} \geq 0$, one for channel. Noting that $\operatorname{Re}\left[\exp \left(-j \mu_{s} c_{1}\right) F\right]=$ $|F| \cos \left(\mu_{s} c_{1}-\operatorname{angle}(F)\right)$, where $F$ is a complex and angle $(F) \in[-\pi, \pi[$ is the angle of $F$, and that $\min _{B>0}\left\{a B^{2}-2 B c\right\}=-c_{+}^{2} / a$, where $a>0$ and $b$ are reals and $x_{+}$is the positive part1 of $x$, then after some manipulations, we obtain

$$
\begin{aligned}
& -\tilde{L}_{h}(\mathbf{c})= \\
& \sum_{s} \frac{1}{\sigma_{s}^{2}} \frac{1}{\sum_{l} w_{h, l, s}}\left|F_{w, h, s}\left(\mu_{s} c_{2}, \mu_{s} c_{3}\right)\right|^{2} \cos _{+}^{2}\left[\mu_{s} c_{1}-\operatorname{angle}\left(F_{w, h, s}\left(\mu_{s} c_{2}, \mu_{s} c_{3}\right)\right)\right],
\end{aligned}
$$

where $F_{w, h, s}\left(\mu c_{2}, \mu c_{3}\right)$ is the windowed/weighted Fourier transform of $u_{s}$,

$$
F_{w, h, s}\left(\omega_{2}, \omega_{3}\right)=\sum_{l} w_{h, l, s} u_{s}\left(x+x_{l}, y+y_{l}\right) \exp \left(-j\left(\omega_{2} x_{l}+\omega_{3} y_{l}\right)\right),
$$

calculated for the frequencies $\left(\omega_{2}=\mu_{s} c_{2}, \omega_{3}=\mu_{s} c_{3}\right)$.

The phase estimate is based on the solution of the optimization of $\tilde{L}_{h}$ over the three phase variables $c_{1}, c_{2}, c_{3}$

$$
\hat{\mathbf{c}}=\arg \max _{\mathbf{c}} \tilde{L}_{h}(\mathbf{c}) .
$$

Let the condition (2) be fulfilled and $Q=\prod q_{s}$. Given fixed values of $c_{2}$ and $c_{3}$, the criterion (9) is a periodic function of $c_{1}$ with the period $2 \pi Q$. Define the main interval for $c_{1}$ to be $[-\pi Q, \pi Q)$. Thus the optimization on $c_{1}$ is restricted to the interval $[-\pi Q, \pi Q)$. We term this effect periodization of the absolute phase $\varphi$, given that its estimation is restricted to this interval only. Because $Q \geq \max _{s} q_{s}$, this periodization means also a partial unwrapping of the phase from the periods $q_{s}$ to the larger period $Q$.

\footnotetext{
${ }^{1}$ I.e., $x_{+}=x$ if $x \geq 0$ and $x_{+}=0$ if $x<0$.
} 


\section{Approximating the ML Estimate}

The 3D optimization (11) is quite demanding. Pragmatically, we compute a suboptimal solution based on the assumption

$$
F_{w, h, s}\left(\hat{c}_{2, s}, \hat{c}_{3, s}\right) \simeq F_{w, h, s}\left(\mu_{s} \hat{c}_{2}, \mu_{s} \hat{c}_{3}\right),
$$

where $\hat{c}_{2}$ and $\hat{c}_{3}$ are the solution of (11) and

$$
\left(\hat{c}_{2, s}, \hat{c}_{3, s}\right) \equiv \arg \max _{c_{2}, c_{3}}\left|F_{w, h, s}\left(c_{2}, c_{3}\right)\right| \text {. }
$$

We note that the assumption (12) holds true at least in two scenarios: a) single channel; b) high signal-to-noise ratio. When the noise power increases, the above assumption is violated and we can not guarantee a performance close to optimal. Nevertheless, we have obtained very good estimates, even in medium to low signal-to-noise ratio scenarios. The comparison between the optimal and suboptimal estimates is, however, beyond the scope of this paper.

Let us introduce the right hand side of (12) into (9). We are then led to the absolute phase estimate $\hat{\varphi}=\hat{c}_{1}$ calculated by the single-variable optimization

$$
\begin{aligned}
& \hat{c}_{1}=\arg \max _{c_{1}} \tilde{L}_{h}\left(c_{1}\right), \\
& \tilde{L}_{h}\left(c_{1}\right)=\sum_{s} \frac{1}{\sigma_{s}^{2}} \frac{1}{\sum_{l} w_{h, l, s}}\left|F_{w, h, s}\left(\hat{c}_{2, s}, \hat{c}_{3, s}\right)\right|^{2} \cos _{+}^{2}\left(\mu_{s} c_{1}-\hat{\psi}_{s}\right) \\
& \hat{\psi}_{s}=\operatorname{angle}\left(F_{w, h, s}\left(\hat{c}_{2, s}, \hat{c}_{3, s}\right)\right) .
\end{aligned}
$$

Phases $\hat{\psi}_{s}$, for $s=1, \ldots, L$, are the LPA estimates of the corresponding wrapped phases $\psi_{s}=W\left(\mu_{s} \varphi\right)$. Again note that the criterion $\tilde{L}_{h}\left(c_{1}\right)$ is periodic with respect to $c_{1}$ with period $2 \pi Q$. Thus, the optimization can be performed only on the finite interval $[-\pi Q, \pi Q)$ :

$$
\hat{c}_{1}=\arg \max _{c_{1} \in[-\pi Q, \pi Q)} \tilde{L}_{h}\left(c_{1}\right) .
$$

If this interval covers the variation range of the absolute phase $\varphi, \varphi \in$ $[-\pi Q, \pi Q)$, the estimate (15) gives a solution of the multifrequency phase unwrap problem. If $\varphi \notin[-\pi Q, \pi Q)$, i.e., the range of the absolute phase $\varphi$ is larger than $2 \pi Q$, then $\hat{c}_{1}$ gives the partial phase unwrapping periodized to the interval $[-\pi Q, \pi Q)$. A complete unwrapping is obtained by applying one of the standard unwrapping algorithms, as these partially unwrapped data can be treated as obtained from a single sensor modulo- $2 \pi Q$ wrapped phase. The above formulas define what we call ML-MF-PEARLS algorithm as short for Maximum Likelihood Multi-Frequency Phase Estimation using Adaptive Regularization based on Local Smoothing.

\section{$5 \quad$ Experimental Results}

Let us we consider a two-frequency scenario with the wavelength $\lambda_{1}<\lambda_{2}$ and compare it versus a single frequency reconstructions with the wavelengths $\lambda_{1}$ 


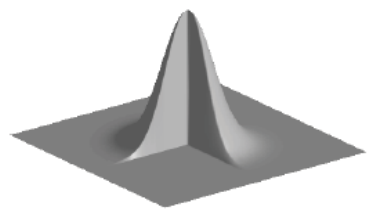

a)

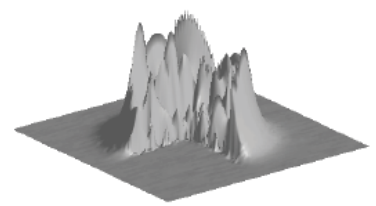

d)

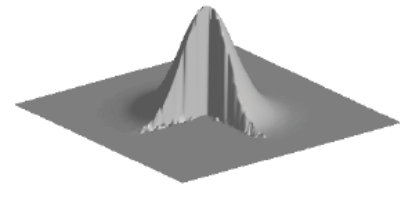

b)

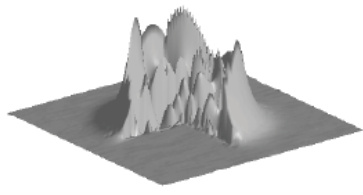

e)

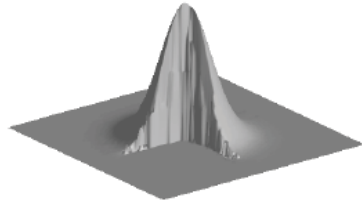

c)

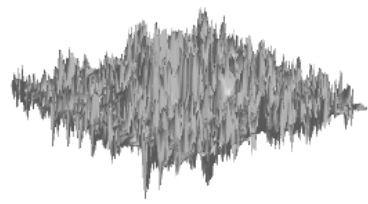

f)

Fig. 1. Discontinuos phase reconstruction: a) true phase surface, b) ML-MF-PEARS reconstruction, $\left(\mu_{1}=1, \mu_{2}=4 / 5\right)$, c) ML-MF-PEARS reconstruction, $\left(\mu_{1}=1, \mu_{2}=\right.$ $9 / 10)$, d) a single frequency PEARLS reconstruction, $\mu_{1}=1$ e) a single frequency PEARLS reconstruction, $\mu_{2}=9 / 10$, f) a single beat-frequency PEARLS reconstruction, $\mu_{12}=10$

and $\lambda_{2}$ as well as versus the synthetic wavelength $\Lambda_{1,2}=\lambda_{1} \lambda_{2} /\left(\lambda_{2}-\lambda_{1}\right)$. The measurement sensitivity is reduced when one considers larger wavelengths. This effect can be modelled by the noise standard deviation proportional to the wavelength. Thus, the noise level in the data corresponding to the wavelength $\Lambda_{1,2}$ is much larger than that for the smaller wavelength $\lambda_{1}$ and $\lambda_{2}$.

The proposed algorithm shows a much better accuracy for the two-frequency data than for the data above mentioned corresponding single frequency scenarios. Another advantage of the multifrequency scenario is its ability to reconstruct the absolute phase for continuous surfaces with huge range and large derivatives. The multifrequency estimation implements an intelligent use of the multichannel data leading to effective phase unwrapping in scenarios in which the unwrapping based on any of the data channels would fail. Moreover, the multifrequency data processing allows to successfully unwrap discontinuous surfaces in situations in which the separate channel processing has no chance for success.

In what follows, we present several experiments illustrating the ML-MFPEARLS performance for continuous and discontinuous phase surfaces. For the phase unwrap of the filtered wrapped phase, we use the PUMA algorithm [1, which is able to work with discontinuities. LPA is exploited with the uniform square windows $w_{h}$ defined on the integer symmetric grid $\{(x, y):|x|,|y| \leq h\}$; thus, the number of pixels of $w_{h}$ is $(2 h+1)$. The ICI parameter was set to $\Gamma=2.0$ and the window sizes to $H \in\{1,2,3,4\}$. The frequencies (13) were computed via FFT zero-padded to the size $64 \times 64$.

As a test function, we use $\varphi(x, y)=A_{\varphi} \times \exp \left(-x^{2} /\left(2 \sigma_{x}^{2}\right)-y^{2} /\left(2 \sigma_{y}^{2}\right)\right)$, a Gaussian shaped surface, with $\sigma_{x}=10, \sigma_{y}=15$, and $A_{\varphi}=40 \times 2 \pi$. The surface is defined on a square grid with integer arguments $x, y,-49 \leq x, y \leq 50$. The 
maximum value of $\varphi$ is $40 \times 2 \pi$ and the maximum values of the first differences are about 15.2 radians. With such high phase differences, any single channel based unwrapping algorithm fail due to many phase differences larger than $\pi$. The noisy observations were generated according to (4), for $B_{s}=1$.

We produce two groups of experiments assuming that we have two channels observations with $\left(\mu_{1}=1, \mu_{2}=4 / 5\right)$ and $\left(\mu_{1}=1, \mu_{2}=9 / 10\right)$, respectively. Then for the synthetic wavelength $\Lambda_{1,2}$ we introduce the phase scaling factor as $\mu_{1,2}=1 / \Lambda_{1,2}=\lambda_{1}-\lambda_{2}$. For the selected $\mu_{1}=1$ and $\mu_{2}=4 / 5$ we have $\mu_{1,2}=1 / 5$ or $\Lambda_{1,2}=5$, and for $\mu_{1}=1$ and $\mu_{2}=9 / 10$ we have $\mu_{1,2}=1 / 10$ or $\Lambda_{1,2}=10$. Note that for all these cases we have the period $Q$ equal to the corresponding beat wavelength $\Lambda_{1,2}=5,10$.

It order to make comparable the accuracy results obtained for the signals of different wavelength, we assume that the noise standard deviation is proportional to the wavelength or inverse proportional to the phase scalling factors $\mu$ :

$$
\sigma_{1}=\sigma / \mu_{1}, \sigma_{2}=\sigma / \mu_{2}, \sigma_{1,2}=\sigma / \mu_{1,2}
$$

where $\sigma$ is a varying parameter. Tables 1 and 2 shows some of the results. The ML-MF-PEARLS shows systematically better accuracy and manage to unwrap the phase when single frequency algorithms fail.

Table 1. RMSE (in rad), $A_{\varphi}=40 \times 2 \pi, \mu_{1}=1, \mu_{2}=4 / 5$

\begin{tabular}{|l|l|l|l|}
\hline Algorithm $\backslash \sigma$ & .3 & .1 & .01 \\
\hline PEARLS, $\mu_{1}=1$ & fail & fail & fail \\
\hline PEARLS, $\mu_{2}=4 / 5$ & fail & fail & fail \\
\hline PEARLS, $\mu_{1,2}=1 / 5$ & fail & 0.722 & 0.252 \\
\hline ML-MF-PEARLS & 0.587 & 0.206 & 0.194 \\
\hline
\end{tabular}

Table 2. RMSE (in $\mathrm{rad}$ ), $A_{\varphi}=40 \times 2 \pi, \mu_{1}=1, \mu_{2}=9 / 10$

\begin{tabular}{|l|l|l|l|}
\hline Algorithm $\backslash \sigma$ & .3 & .1 & .01 \\
\hline PEARLS, $\mu_{1}=1$ & fail & fail & fail \\
\hline PEARLS, $\mu_{2}=9 / 10$ & fail & fail & fail \\
\hline PEARLS, $\mu_{1,2}=1 / 10$ & fail & 3.48 & 0.496 \\
\hline ML-MF-PEARLS & 1.26 & 0.204 & 0.194 \\
\hline
\end{tabular}

We now illustrate the potential in handling discontinuities of bringing together the adaptive denoising and the unwrapping. For the test, we use the Gaussian surface with one quarter set to zero. The corresponding results are shown in Fig. 1. The developed algorithm confirms its clear ability to reconstruct a strongly varying discontinuous absolute phase from noisy multifrequency data.

Figure 2 shows results based on a simulated InSAR example supplied in the book 3. This data set have been generated based on a real digital elevation model of mountainous terrain around Long's Peak using a high-fidelity InSAR 

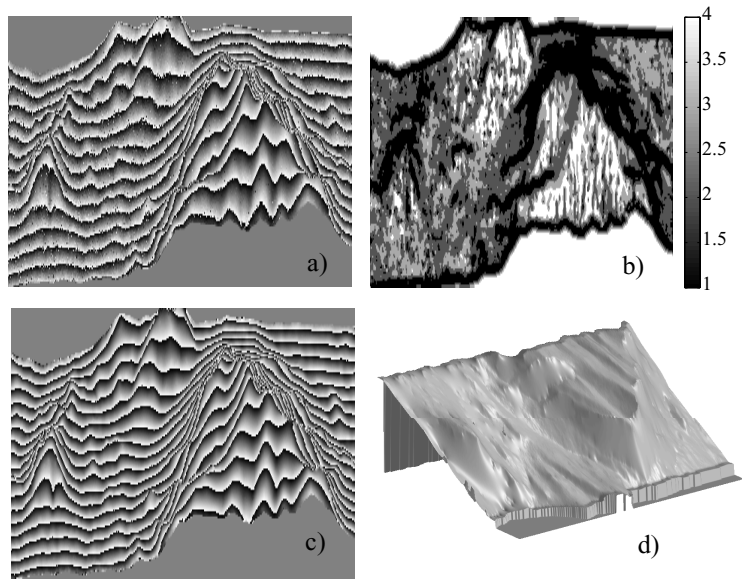

Fig. 2. Simulated SAR based on a real digital elevation model of mountainous terrain around Long's Peak using a high-fidelity InSAR simulator (see 3 for details): a) original interferogram (for $\mu_{1}=1$ ); b) Window sizes given by ICI; c) LPA phase estimation corresponding to $\psi_{1}=W\left(\mu_{1} \varphi\right)$; d) ML-MF-PEARS reconstruction for $\mu_{1}=1$ and $\mu_{2}=4 / 5$ corresponding to $\mathrm{rmse}=0.3 \mathrm{rad}$ (see text for details)

simulator that models the SAR point spread function, the InSAR geometry, the speckle noise (4 looks) and the layover and shadow phenomena. To simulate diversity in the acquisition, besides the interferogram supplied with the data, we have generated another interferogram, according to the statistics of a fully developed speckle (see, e.g., [7] for details) with a frequency $\mu_{2}=4 / 5$.

Figure 2 a) shows the original interferogram corresponding to $\mu_{1}=1$. Due to noise, areas of low coherence, and layover, the estimation of the original phase based on this interferogram is a very hard problem, which does not yield reasonable estimates, unless external information in the form of quality maps is used [3, 7]. Parts b) and c) shows the window sizes given by ICI and the LPA phase estimation corresponding to $\psi_{1}=W\left(\mu_{1} \varphi\right)$, respectively. Part d) shows ML-MF-PEARS reconstruction, where the areas of very low coherence were removed and interpolated from the neighbors. We stress that we have not used these quality information in the estimation phase. The estimation error is RMSE $=0.3 \mathrm{rad}$, which, having in mind that the phase range is larger the 120 $\mathrm{rad}$, is a very good figure.

The leading term of the computational complexity of the ML-MF-PEARLS is $O\left(n^{2.5}\right)$ ( $n$ is the number of pixels) due to the PUMA algorithm. This is, however, the worst case figure. The practical complexity is very close to $O(n)$ 1. In practice, we have observed that a good approximation of the algorithm complexity is given by complexity of $n L$ FFTs, i.e., $\left(2 L P^{2} \log _{2} P\right) n$, where $L$ is the number of channels and $P \times P$ is the size of the FFTs. The examples shown is this section took less than 30 seconds in a $\mathrm{PC}$ equipped with a dual core $\mathrm{CPU}$ running at $3.0 \mathrm{GHz}$ 


\section{Concluding Remarks}

We have introduced ML-MF-PEARLS, a new adaptive algorithm to estimate the absolute phase from frequency diverse wrapped observations. The new methodology is based on local maximum likelihood phase estimates. The true phase is approximated by a local polynomial with varying adaptive neighborhood used in reconstruction. This mechanism is critical in preserving the discontinuities of piecewise smooth absolute phase surfaces. The ML-MF-PEARLS, algorithm, besides filtering the noise, yields a $2 \pi Q$-periodic solution, where $Q>1$ is an integer. Depending on the value of $Q$ and of the original phase range, we may obtain complete or partial phase unwrapping. In the latter case, we apply the recently introduced robust (in the sense of discontinuity preserving) PUMA unwrapping algorithm [1. In a set of experiments, we gave evidence that the ML-MFPEARLS algorithm is able to produce useful unwrappings, whereas state-of-the art competitors fail.

\section{Acknowledgments}

This research was supported by the "Fundação para a Ciência e Tecnologia", under the project PDCTE/CPS/49967/2003, by the European Space Agency, under the project ESA/C1:2422/2003, and by the Academy of Finland, project No. 213462 (Finnish Centre of Excellence program 2006 - 2011).

\section{References}

1. Bioucas-Dias, J., Valadão, G.: Phase unwrapping via graph cuts. IEEE Trans. Image Processing 16(3), 684-697 (2007)

2. Graham, L.: Synthetic interferometer radar for topographic mapping. Proceeding of the IEEE 62(2), 763-768 (1974)

3. Ghiglia, D., Pritt, M.: Two-Dimensional Phase Unwrapping. In: Theory, Algorithms, and Software. John Wiley \& Sons, New York (1998)

4. Zebker, H., Goldstein, R.: Topographic mapping from interferometric synthetic aperture radar. Journal of Geophysics Research 91(B5), 4993-4999 (1986)

5. Patil, A., Rastogi, P.: Moving ahead with phase. Optics and Lasers in Engineering 45, 253-257 (2007)

6. Goldstein, R., Zebker, H., Werner, C.: Satellite radar interferometry: Twodimensional phase unwrapping. In: Symposium on the Ionospheric Effects on Communication and Related Systems. Radio Science, vol. 23, pp. 713-720 (1988)

7. Bioucas-Dias, J., Leitao, J.: The $Z \pi M$ algorithm: a method for interferometric image reconstruction in SAR/SAS. IEEE Trans. Image Processing 11(4), 408-422 (2002)

8. Yun, H.Y., Hong, C.K., Chang, S.W.: Least-square phase estimation with multiple parameters in phase-shifting electronic speckle pattern interferometry. J. Opt. Soc. Am. A 20, 240-247 (2003)

9. Kemao, Q.: Two-dimensional windowed Fourier transform for fringe pattern analysis: principles, applications and implementations. Opt. Lasers Eng. 45, 304-317 (2007) 
10. Katkovnik, V., Astola, J., Egiazarian, K.: Phase local approximation (PhaseLa) technique for phase unwrap from noisy data. IEEE Trans. on Image Processing 46(6), 833-846 (2008)

11. Katkovnik, V., Egiazarian, K., Astola, J.: Local Approximation Techniques in Signal and Image Processing. SPIE Press, Bellingham (2006)

12. Servin, M., Marroquin, J.L., Malacara, D., Cuevas, F.J.: Phase unwrapping with a regularized phase-tracking system. Applied Optics 37(10), 1917-1923 (1998)

13. Pascazio, V., Schirinzi, G.: Multifrequency InSAR height reconstruction through maximum likelihood estimation of local planes parameters. IEEE Transactions on Image Processing 11(12), 1478-1489 (2002)

14. Servin, M., Cuevas, F.J., Malacara, D., Marroguin, J.L., Rodriguez-Vera, R.: Phase unwrapping through demodulation by use of the regularized phase-tracking technique. Appl. Opt. 38, 1934-1941 (1999)

15. Servin, M., Kujawinska, M.: Modern fringe pattern analysis in interferometry. In: Malacara, D., Thompson, B.J. (eds.) Handbook of Optical Engineering, ch. 12, pp. 373-426, Dekker (2001)

16. Born, M., Wolf, E.: Principles of Optics, 7th edn. Cambridge University Press, Cambridge (2002)

17. Xia, X.-G., Wang, G.: Phase unwrapping and a robust chinese remainder theorem. IEEE Signal Processing Letters 14(4), 247-250 (2007)

18. McClellan, J.H., Rader, C.M.: Number Theory in Digital Signal Processing. Prentice-Hall, Englewood Cliffs (1979)

19. Goldreich, O., Ron, D., Sudan, M.: Chinese remaindering with errors. IEEE Trans. Inf. Theory 46(7), 1330-1338 (2000)

20. Bioucas-Dias, J., Katkovnik, V., Astola, J., Egiazarian, K.: Absolute phase estimation: adaptive local denoising and global unwrapping. Applied Optics 47(29), $5358-5369$ (2008) 